\title{
A reduced formulation for pseudoinvex vector functions
}

\author{
Manuel Arana-Jiménez • Riccardo \\ Cambini · Laura Carosi
}

Received: date / Accepted: date

\begin{abstract}
Vector pseudoinvexity is characterized in the current literature by means of a suitable functional which depends on two variables. In this paper, vector pseudoinvexity is characterized by means of a functional which depends on one variable only. For this very reason, the new characterizing conditions are easier to be verified.
\end{abstract}

Keywords Multiobjective optimization · Vector Invexity · Vector pseudoinvexity

Mathematics Subject Classification (2000) 90C29 - 90C26 - 90C46 . $49 \mathrm{~N} 15$

\section{Introduction}

Invexity and its generalizations have been widely studied over the past years occupying a preeminent role in Optimization Theory. Indeed scalar invex functions are the widest class of functions for which Fermat necessary optimality condition becomes also sufficient and this partially explains the great interest in such a class.

With the aim of finding wider classes of functions sharing the nice properties of invex functions, different notions of generalized invexity have been proposed. Most of the new suggested conditions involve more and more parameters and/or functionals. In some cases the new classes involve conditions which

Manuel Arana-Jiménez

Department of Statistics and Operational Research, Faculty of SSCC and Communication, University of Cádiz, Av. de la Universidad, 11405-Jerez, Spain Tel.: +34956037875.

E-mail: manuel.arana@uca.es

Riccardo Cambini · Laura Carosi

Department of Economics and Management, University of Pisa, Via Ridolfi 10, 56124, Pisa Italy

E-mail: riccardo.cambini@unipi.it

E-mail: laura.carosi@unipi.it 
are not so easy to be verified. Furthermore, it is not sometimes clear whether the new proposed classes of generalized invex functions are real generalizations of the existing ones or they simply coincide with some of them (see also Zălinescu (2014) and (2016)).

The study of invexity concepts has been done for both scalar and vector case (see for example Mishra and Giorgi [11] , Pini and Singh [14] and reference therein). In the scalar case, it has been proved by Craven and Glover [9] that the notions of invexity and pseudoinvexity coincide and the same happens for other "new" classes of "generalized invex" functions (see for example Caprari [6]). Moving towards the vector case, invexity and pseudoinvexity do not longer coincide and pseudoinvexity is the largest class for which critical points are efficient points (see for example Craven [7], Osuna-Gómez et al. [12] and Arana-Jiménez et al. [2]). Unlike the latest trends of the recent literature, the aim of this paper is to state characterizations involving "simplified" parameter functionals and therefore to obtain necessary and sufficient conditions which are easier to be verified than the existing ones. While the classical notions of pseudoinvexity are given by means of parameter functionals depending on two variables, the proposed characterizations are based on parameter functionals depending on one variable only. As in Arana-Jiménez et al. [2] and following the notations introduced by Cambini [5] for generalized convexity, vector pseudoinvexity is defined by using two order relations induced by $\operatorname{Int} C$, namely the interior of a convex, closed, pointed cone $C$, and by $C^{0}$, namely the cone $C$ without the origin. It's worth recalling that similar notations have been used also in Gutiérrez et al. [10] where vector locally Lipschitz functions are considered.

\section{Vector pseudoinvexity: definitions and preliminary results}

It is well-known that pseudoinvexity concepts are based on the use of parameter functionals $\eta(\cdot, \cdot)$ depending on two variables. Following Arana-Jiménez et al. [2], in this paper the definitions of vector invexity and pseudoinvexity are given with respect to a closed convex cone $C$ with nonempty interior. The notion of scalar pseudoinvexity can be extended to the vector case in several different ways. The different definitions are obviously related to how the relation " $\leq$ " for real numbers can be extended in $\mathbb{R}^{p}$ with $p>1$. In what follows two classes of pseudoinvex are considered; as it will be later specified, each of them is useful in studying a specific efficiency property.

Definition 1 Let $f: X \rightarrow \mathbb{R}^{p}$, with $X \subseteq \mathbb{R}^{n}$ open set, be a differentiable vector valued function and let $C \subseteq \mathbb{R}^{p}$ be a closed convex pointed cone with nonempty interior. The function $f$ is said to be

i) invex if there exists a vector function $\eta: X \times X \rightarrow \mathbb{R}^{n}$ such that for all $x, \bar{x} \in X$

$$
f(x)-f(\bar{x}) \in J_{f}(\bar{x}) \eta(x, \bar{x})+C ;
$$


where $J_{f}(\bar{x})$ is the Jacobian matrix of function $f$ evaluated at $\bar{x}$.

ii) (Int $C$, Int $C$ )-pseudoinvex if there exists a vector function $\eta: X \times X \rightarrow \mathbb{R}^{n}$ such that for all $x, \bar{x} \in X$

$$
f(x)-f(\bar{x}) \in-\operatorname{Int} C \Rightarrow J_{f}(\bar{x}) \eta(x, \bar{x}) \in-\operatorname{Int} C ;
$$

iii) $\left(C^{0}, \operatorname{Int} C\right)$-pseudoinvex if there exists a vector function $\eta: X \times X \rightarrow \mathbb{R}^{n}$ such that for all $x, \bar{x} \in X$

$$
f(x)-f(\bar{x}) \in-C^{0} \Rightarrow J_{f}(\bar{x}) \eta(x, \bar{x}) \in-\operatorname{Int} C .
$$

Referring to the current literature, Gutiérrez et al. [10] introduced the notions of $(\operatorname{Int} C, \operatorname{Int} C)$-pseudoinvexity-I and $(\operatorname{Int} C, \operatorname{Int} C)$-pseudoinvexity-II for locally Lipschutz functions. In the differentiable case these two concepts coincide with ii) of Definition 1. Similarly, Gutiérrez et al. [10] define $\left(C^{0}, \operatorname{Int} C\right)$ pseudoinvexity-I and $\left(C^{0}, \operatorname{Int} C\right)$-pseudoinvexity-II and in the differentiable case they both coincide with $\left(C^{0}, \operatorname{Int} C\right)$-pseudoinvexity.

When $C$ is the Pareto cone, in Arana-Jiménez et al. [1], $(\operatorname{Int} C, \operatorname{Int} C)$-pseudoinvex functions have been called "pseudoinvex-I", while $\left(C^{0}, \operatorname{Int} C\right)$-pseudoinvex functions have been called "pseudoinvex-II". Still remaining in the paretian case, in Craven [8] and Osuna-Gómez et al. [12], $(\operatorname{Int} C, \operatorname{Int} C)$-pseudoinvex functions are simply called pseudoinvex functions. With respect to the non-differentiable case, the reader can see Gutierréz et al. [10].

Regarding the relationships between i), ii) and iii) in Definition 1, the following inclusions are straightforward:

i) if a function is invex, then it is $(\operatorname{Int} C, \operatorname{Int} C)$-pseudoinvex;

ii) if a function is $\left(C^{0}, \operatorname{Int} C\right)$-pseudoinvex, then it is $(\operatorname{Int} C, \operatorname{Int} C)$-pseudoinvex.

The following functions underline that the above inclusion relationships are proper and that none of the considered classes of functions is empty.

1. $f=\left(f_{1}, f_{2}\right): \mathbb{R} \rightarrow \mathbb{R}^{2}$, with $f(x)=\left(x^{2}, 5\right)$ and $C=\mathbb{R}_{+}^{2} \cdot f$ is convex and hence $f$ is invex with $\eta(x, \bar{x})=(x-\bar{x})$. On the other hand, for $\bar{x}=0$ it is $J_{f}(\bar{x})=[0,0]^{T}$, so that there exists no $\eta(x, \bar{x})$ such that $J_{f}(\bar{x}) \eta(x, \bar{x}) \in$ $-\operatorname{Int} C$. Consequently, $f$ is not $\left(C^{0}, \operatorname{Int} C\right)$-pseudoinvex.

2. $f=\left(f_{1}, f_{2}\right): \mathbb{R} \rightarrow \mathbb{R}^{2}$, with $f(x)=\left(x^{2},-x^{2}\right)$ and $C=\mathbb{R}_{+}^{2}$. Since $f(x)-$ $f(\bar{x}) \notin C^{0}$ for all $x, \bar{x} \in \mathbb{R}, f$ is trivially $\left(C^{0}, \operatorname{Int} C\right)$-pseudoinvex. On the other hand, take $\bar{x}=0$ it is $J_{f}(\bar{x})=[0,0]^{T}$ and $f(x)-f(0)=\left[x^{2},-x^{2}\right]^{T}$. Consequently there exists no $\eta(x, \bar{x})$ such that $\left[x^{2},-x^{2}\right]^{T} \in[0,0]^{T} \eta(x, \bar{x})+$ $C$ and hence $f$ is not invex.

3. $f=\left(f_{1}, f_{2}\right): \mathbb{R} \rightarrow \mathbb{R}^{2}$, with $f(x)=\left(-x^{2}, 5\right)$ and $C=\mathbb{R}_{+}^{2}$. With a similar arguments of 1 . and 2 . it can be easily verified that $f$ is $(\operatorname{Int} C, \operatorname{Int} C)$ pseudoinvex, but it is neither $\left(C^{0}, \operatorname{Int} C\right)$-pseudoinvex nor invex.

As it will be pointed out in the next section, the different notions of pseudoinvex are related to different concepts of efficiency; for the sake of completeness let us recall the following definitions of efficient points for vector valued problems where the partial ordering in the image space is induced by a closed convex pointed cone with nonempty interior. 
Definition 2 Let $f: X \rightarrow \mathbb{R}^{p}$, with $X \subseteq \mathbb{R}^{n}$ open set, be a differentiable vector valued function and let $C \subseteq \mathbb{R}^{p}$ be a closed convex pointed cone with nonempty interior and let $C^{+}=\left\{\lambda \in \mathbb{R}^{p}\right.$ such that $\left.\lambda^{T} c \geq 0, \forall c \in C\right\} \subset \mathbb{R}^{p}$ be the positive polar cone of $C$. A point $\bar{x} \in X$ is

i) a weakly efficient solution for $f$ on $X$ if $\nexists x \in X$ such that $f(x) \in f(\bar{x})-$ Int $C$, that is to say that $(f(X)-f(\bar{x})) \cap(-\operatorname{Int} C)=\emptyset$;

ii) an efficient solution for $f$ on $X$ if $\nexists x \in X$ such that $f(x) \in f(\bar{x})-C^{0}$, that is to say that $(f(X)-f(\bar{x})) \cap(-C)=\{0\}$;

iii) a $C$-stationary point for $f$ if there exists $\lambda \in C^{+}, \lambda \neq 0$, such that $\lambda^{T} J_{f}(\bar{x})=0$.

\section{A new characterization for pseudoinvex vector functions}

The aim of this section is twofold: to prove that pseudoinvexity can be expressed by means of a parameter functional depending on just one variable and to recall that this property characterizes the efficiency of stationary points (see Arana-Jiménez et al. [2]). This result will be proved by means of the following Gordan-type result whose proof is given for the sake of completeness.

Lemma 1 Let $A \in \mathbb{R}^{p \times n}$ be a matrix and let $C \subseteq \mathbb{R}^{p}$ be a closed convex pointed cone with nonempty interior.

If

$$
\lambda^{T} A \neq 0 \quad \forall \lambda \in C^{+}, \lambda \neq 0,
$$

then

$$
\exists v \in \mathbb{R}^{n} \text { such that } A v \in-\text { IntC. }
$$

Proof Assume by contradiction that:

$$
A v \notin-\operatorname{Int} C \quad \forall v \in \mathbb{R}^{n} .
$$

By using the notation $W=\left\{w \in \mathbb{R}^{p}: w=A v, v \in \mathbb{R}^{n}\right\}$ such an assumption can be rewritten as $(-\operatorname{Int} C) \cap W=\emptyset$. Since $W$ and $-\operatorname{Int} C$ are convex sets, then from Theorem 11.3 in [15] there exists an hyperplane which properly separates -IntC and $W$. Therefore, since $W$ is a cone, from Theorem 11.7 in [15] there exists a separating hyperplane containing the origin, that is

$$
\exists \lambda \in \mathbb{R}^{p} \text {, s.t. } \lambda^{T}(-c) \leq 0 \leq \lambda^{T} w \quad \forall c \in \operatorname{Int} C \forall w \in W .
$$

Consequently

$$
\exists \lambda \in C^{+}, \lambda \neq 0, \text { such that } \lambda^{T} w \geq 0 \forall w \in W .
$$

This implies that $\lambda^{T} A v \geq 0 \forall v \in \mathbb{R}^{n}$ which yields $\lambda^{T} A=0$. This contradicts the hypothesis so that the result is proved.

It is now possible to provide new characterizations of vector pseudoinvexity. 
Theorem 1 Let $f: X \rightarrow \mathbb{R}^{p}$, with $X \subseteq \mathbb{R}^{n}$ open set, be a differentiable vector valued function and let $C \subseteq \mathbb{R}^{p}$ be a closed convex pointed cone with nonempty interior. The following conditions are equivalent:

i) there exists a vector function $\mu: X \rightarrow \mathbb{R}^{n}$ such that for all $x, \bar{x} \in X$

$$
f(x)-f(\bar{x}) \in-\operatorname{Int} C \Rightarrow J_{f}(\bar{x}) \mu(\bar{x}) \in-\operatorname{Int} C ;
$$

ii) $f$ is (IntC, IntC)-pseudoinvex;

iii) any stationary points of $f$ is a weakly efficient solution for $f$ on $X$.

Proof $i) \Rightarrow$ ii) The result follows trivially by choosing $\eta(x, \bar{x})=\mu(\bar{x})$.

ii $\Rightarrow$ iii) Assume on the contrary that there exists a stationary point which is not weakly efficient, that is to say that $\exists x, \bar{x} \in X, \exists \lambda \in C^{+}, \lambda \neq 0$, such that $f(x) \in f(\bar{x})-\operatorname{Int} C$ and $\lambda^{T} J_{f}(\bar{x})=0$. From condition ii) it yields $J_{f}(\bar{x}) \eta(x, \bar{x}) \in-\operatorname{Int} C$. Being $\lambda \in C^{+}, \lambda \neq 0$, this implies $\lambda^{T} J_{f}(\bar{x}) \eta(x, \bar{x})<0$ so that $\lambda^{T} J_{f}(\bar{x}) \neq 0$ which is a contradiction.

iii) $\Rightarrow$ i) For all $\bar{x} \in X$ let us consider the set:

$$
S(\bar{x})=\{x \in X: f(x)-f(\bar{x}) \in-\operatorname{Int} C\} .
$$

Let us preliminarily state that if $S(\bar{x}) \neq \emptyset$ then $\exists v(\bar{x}) \in \mathbb{R}^{n}$ such that $J_{f}(\bar{x}) v(\bar{x}) \in-\operatorname{Int} C$. With this aim, notice that if $S(\bar{x}) \neq \emptyset$ then $\bar{x}$ is not a weakly efficient solution and hence is not a stationary points, that is to say that $\lambda^{T} J_{f}(\bar{x}) \neq 0 \forall \lambda \in C^{+}, \lambda \neq 0$; by means of Lemma 1 this implies that $\exists v(\bar{x}) \in \mathbb{R}^{n}$ such that $J_{f}(\bar{x}) v(\bar{x}) \in-\operatorname{Int} C$. In order to prove the result let us now define the following parameter functional:

$$
\mu(\bar{x})=\left\{\begin{array}{cc}
0 & \text { if } S(\bar{x})=\emptyset \\
v(\bar{x}) & \text { if } S(\bar{x}) \neq \emptyset
\end{array}\right.
$$

For all $x, \bar{x} \in X$ such that $f(x)-f(\bar{x}) \in-\operatorname{Int} C$ it results $S(\bar{x}) \neq \emptyset$, so that $J_{f}(\bar{x}) \mu(\bar{x})=J_{f}(\bar{x}) v(\bar{x}) \in-\operatorname{Int} C$ which yields the thesis.

The following further result can be proved analogously.

Theorem 2 Let $f: X \rightarrow \mathbb{R}^{p}$, with $X \subseteq \mathbb{R}^{n}$ open set, be a differentiable vector valued function and let $C \subseteq \mathbb{R}^{p}$ be a closed convex pointed cone with nonempty interior. The following conditions are equivalent:

i) there exists a vector function $\mu: X \rightarrow \mathbb{R}^{n}$ such that for all $x, \bar{x} \in X$

$$
f(x)-f(\bar{x}) \in-C^{0} \Rightarrow J_{f}(\bar{x}) \mu(\bar{x}) \in-\operatorname{Int} C ;
$$

ii) $f$ is $\left(C^{0}\right.$, IntC $)$-pseudoinvex;

iii) any stationary points of $f$ is an efficient solution for $f$ on $X$.

The following examples point out that the proposed new characterizations (based on functionals depending on one variable only) offer more operative conditions with respect to the classical ones. This relies on the fact that searching a suitable functional which depends on just one variable $\bar{x}$ is an easier task than looking for a functional which depends on two variables, that is $x, \bar{x}$. 
Example 1 Let us consider the function $f=\left(f_{1}, f_{2}\right): \mathbb{R} \rightarrow \mathbb{R}^{2}$, with

$$
f(x)=\left(f_{1}(x), f_{2}(x)\right)=\left(x^{3}+1, x^{2}+10\right)
$$

and let $C=\mathbb{R}_{+}^{2}$ be the Pareto Cone.

It can be proved that $f$ is $(\operatorname{Int} C, \operatorname{Int} C)$-pseudoinvex. Referring to Theorem 1 , let us consider the functional $\mu(\bar{x})=-\bar{x}^{3}$; it can be easily seen that for any $x, \bar{x}$ such that $f(x)-f(\bar{x}) \in-\operatorname{Int} C$ it results $J_{f}(\bar{x}) \mu(\bar{x}) \in-\operatorname{Int} C$. In this light first notice that:

$$
J_{f}(\bar{x}) \mu(\bar{x})=\left[\begin{array}{c}
3 \bar{x}^{2} \\
2 \bar{x}
\end{array}\right]\left(-\bar{x}^{3}\right)=\left[\begin{array}{c}
\left(3 \bar{x}^{2}\right)\left(-\bar{x}^{3}\right) \\
-2 \bar{x}^{4}
\end{array}\right]
$$

When $\bar{x}=0$ it is $f(x)-f(0)=\left(x^{3}, x^{2}\right)$ so that function $f$ verifies (4) since condition $f(x)-f(0) \in-\operatorname{Int} C$ is not satisfied for any $x$. When $\bar{x}>0$ it is $J_{f}(\bar{x}) \mu(\bar{x}) \in-\operatorname{Int} C$ for all $x \in \mathbb{R}$ and hence $f$ verifies (4). Let finally be $\bar{x}<0$; from $f_{1}(x)-f_{1}(\bar{x})<0$ and from the strict increaseness of $f_{1}$ it follows that $x<\bar{x}$; being $\bar{x}<0$, it yields $x^{2}>\bar{x}^{2}$ so that $f_{2}(x)-f_{2}(\bar{x})>0$; as a consequence condition $f(x)-f(\bar{x}) \in-\operatorname{Int} C$ is not satisfied for any $\bar{x}<0$ and hence $f$ trivially verifies (4). Furthermore, let us prove that $f$ is not invex. To this purpose, take $\bar{x}=0, x=-2$ and consider any function $\eta: X \times X \rightarrow$ $\mathbb{R}$. Therefore, $J_{f}(0)=[0,0]^{T}$, and then $J_{f}(0) \eta(-2,0)=0$. We have that $f(-2)-f(0)=(-8,4)$. Then,

$$
f(-2)-f(0)=(-8,4) \notin C=J_{f}(0) \eta(-2,0)+C,
$$

what implies that condition (1) is not verified for any $\eta$, and then, $f$ is not invex.

Example 2 Let us consider $X=(-2,2)$ and the function $f=\left(f_{1}, f_{2}\right): X \rightarrow$ $\mathbb{R}^{2}$, with

$$
f(x)=\left(f_{1}(x), f_{2}(x)\right)=\left(-\frac{4}{3} x^{3}-2 x,-x^{5}-x\right),
$$

and the cone

$$
C=\left\{\left(x_{1}, x_{2}\right) \in \mathbb{R}^{2}:-10 x_{1}+x_{2} \leq 0, x_{2} \geq 0\right\} .
$$

Notice that

$$
\begin{gathered}
-C^{0}=\left\{\left(x_{1}, x_{2}\right) \in \mathbb{R}^{2}:-10 x_{1}+x_{2} \geq 0, x_{2} \leq 0\left(x_{1}, x_{2}\right) \neq 0\right\}, \\
-\operatorname{Int} C=\left\{\left(x_{1}, x_{2}\right) \in \mathbb{R}^{2}:-10 x_{1}+x_{2}>0, x_{2}<0\right\},
\end{gathered}
$$

Let us prove that $f$ is $\left(C^{0}, \operatorname{Int} C\right)$-pseudoinvex. We have that

$$
J_{f}(\bar{x})=\left[\begin{array}{l}
-4 x^{2}-2 \\
-5 x^{4}-1
\end{array}\right] .
$$


Referring to Theorem 2 , let us consider the functional $\mu(\bar{x})=\bar{x}^{2}+1$; it can be easily seen that for any $x, \bar{x}$ such that $f(x)-f(\bar{x}) \in-C^{0}$ it results $J_{f}(\bar{x}) \mu(\bar{x}) \in$ $-\operatorname{Int} C$. In fact, let us see that $J_{f}(\bar{x}) \mu(\bar{x}) \in-\operatorname{Int} C$ as follows.

$$
J_{f}(\bar{x}) \mu(\bar{x})=\left[\begin{array}{l}
\left(-4 x^{2}-2\right)\left(x^{2}+1\right) \\
\left(-5 x^{4}-1\right)\left(x^{2}+1\right)
\end{array}\right]
$$

Taking into account (6), we have that $J_{f}(\bar{x}) \mu(\bar{x}) \in-\operatorname{Int} C$ if and only if the following conditions hold

$$
\begin{aligned}
& \left(-5 \bar{x}^{4}-1-10\left(-4 \bar{x}^{2}-2\right)\right)\left(\bar{x}^{2}+1\right)>0, \\
& \left(-5 \bar{x}^{4}-1\right)\left(\bar{x}^{2}+1\right)<0 .
\end{aligned}
$$

By means of simple computations we have that conditions (7) are satisfied for all $\bar{x} \in X$. Therefore condition (5) is verified, and from Theorem 2 , it follows that $f$ is $\left(C^{0}, \operatorname{Int} C\right)$-pseudoinvex.

Acknowledgements The authors are very grateful to the anonymous referees for their valuable remarks and suggestions. Furthermore, the authors would like to express their sincere appreciation and gratitude to Professor Laura Martein for her insightful comments and her fruitful support.

\section{References}

1. Arana-Jiménez, M., Rufián-Lizana, A., Osuna-Gómez, R., Ruiz-Garzón, G., A characterization of pseudoinvexity in multiobjective programming. Mathematical and Computer Modelling 48, 1719-1723 (2008).

2. Arana-Jiménez, M., Cambini R., Rufián-Lizana A., C-efficiency in nondifferentiable vector optimization, Mathematical and computer Modelling 57, 1148-1153 (2013).

3. Arana-Jiménez, M., Cambini R., Conic efficiency and duality in nondifferentiable multiobjective mathematical programming, Journal of Nonlinear and Convex Analysis, 16 , 2507-2520 (2015)

4. Ben-Israel, A., Mond, B., What is Invexity?, Journal of Australian Mathematical Society Series B, 28, 1-9 (1986).

5. Cambini, R., Some new classes of generalized concave vector-valued functions, Optimization 36, 11-24 (1996).

6. Caprari, E., $\rho$-invex functions and $(F, \rho)$-convex functions: properties and equivalencies, Optimization 52, 65-74 (2003)

7. Craven, B. D., Kinds of vector invex, Taiwanese Journal of Mathematics 14, 1925-1933, (2010).

8. Craven, B.D., Global invexity and duality in mathematical programming, Asia-Pacific Journal of Operational Research 19, 169-175 (2002).

9. Craven, B.D., Glover, B.M., Invex Functions and Duality, Journal of Australian Mathematical Society Series A 39, 1-20 (1985).

10. Gutiérrez, C., Jiménez, B., Novo, V., Ruiz-Garzón, G., Efficiency through variationallike inequalities with Lipschitz functions, Applied Mathematics and Computation 259, 438-449 (2015).

11. Mishra, S.K., Giorgi, G., Invexity and Optimization, Nonconvex Optimization and its Applications, Springer, Berlin (2008).

12. Osuna-Gómez, R., Beato-Moreno, A., Rufián-Lizana A., Generalized convexity in multiobjective programming, Journal of Mathematical Analysis and Applications, 233, 205-220, (1999). 
13. Osuna-Goméz, R., Rufián-Lizana, A., Ruiz-Canales, P., Invex functions and generalized convexity in multiobjective programming, Journal of Optimization Theory and Applications, 98, 651-661 (1998).

14. Pini, R., Singh, C., A survey of recent[1985-1995]advances in generalized convexity with applications to duality theory and optimality conditions, Optimization 39, 311-360 (1997).

15. Rockafellar R.T., (1970), Convex Analysis, Princeton University Press.

16. Zălinescu, C., (2014), "A critical view on invexity." Journal of Optimization Theory and Applications 162, 695-704, (2014).

17. Zălinescu, C., (2016) "On V. Latorre and D.Y. Gao's paper 'Canonical duality for solving general nonconvex constrained problems', Optimization Letters: 1-7. 